tung der Medien in Liechtenstein immer wieder leicht herauszufinden ist, um welche Person es sich handelt. Stattdessen sollte unserer Ansicht nach primär die Sache im Vordergrund stehen.

Außerdem äußerten wir uns zu umfassenden Fragen des Datenschutzes am Arbeitsplatz.

Im Zusammenhang mit der Vorratsdatenspeicherung waren wir weiterhin aktiv. So wiesen wir neben dem genannten Vortrag die Regierung darauf hin, dass gemäß der Rechtsprechung des deutschen Bundesverfassungsgerichts, aber auch gemäß der entsprechenden Richtlinie, ein Anpassungsbedarf der Strafprozessordnung besteht.

Im Rahmen unserer internationalen Aufgaben fand die Schengen-Evaluation statt. Liechtenstein ist seit Ende des letzten Jahres Schengen-Mitglied. Dies bedeutet für uns, dass wir insbesondere regelmäßige Kontrollen durchführen müssen. Dies wurde bei der Evaluation gefordert.

Wir nahmen zu 24 Gesetzesvorhaben Stellung. Darunter befand sich im Rahmen einer neuerlichen Revision des Datenschutzgesetzes (DSG), aber auch etwa die Revision der Strafprozessordnung, die durch die Umsetzung des Rahmenbeschlusses zum Datenschutz in der Dritten Säule notwendig wurde, oder die Gesamtrevision des Versicherungsaufsichtsgesetzes.

Für den Einsatz für die Belange der Privatsphäre und die aktive Unterstützung der Regierung, des Landtags und der Landesverwaltung bedankte sich der Datenschutzbeauftragte ausdrücklich.

Der Bericht steht auf der Website http://www.IIv.li/pdf-Ilv-dss-taetigkeitsbericht_2011.pdf

zur Verfügung.

\section{Konferenz der Datenschutzbeauftragten des Bundes und der Länder: Entschließung vom 23. Mai 2012}

Patientenrechte müssen umfassend gestärkt werden Mit dem im Januar 2012 der Öffentlichkeit vorgestellten und nun dem Bundeskabinett zugeleiteten Entwurf eines Gesetzes zur Verbesserung der Rechte von Patientinnen und Patienten (Patientenrechtegesetz) sollen insbesondere die bislang von den Gerichten entwickelten Grundsätze des Arzthaftungs- und Behandlungsrechts zusammengeführt und transparent für alle an einer Behandlung Beteiligten geregelt werden.

Die Konferenz der Datenschutzbeauftragten des Bundes und der Länder teilt das Anliegen der Bundesregierung, die Rechte von Patientinnen und Patienten zu stärken.

Die Datenschutzkonferenz hält allerdings die vorgelegten Regelungen in dem Entwurf eines Patientenrechtegesetzes für nicht ausreichend. Sie fordert die Bundesregierung nachdrücklich auf, den Gesetzentwurf zu überarbeiten und dabei die folgenden Aspekte zu berücksichtigen:

- Die vertraglichen Offenbarungsobliegenheiten der Patientinnen und Patienten gegenüber den Behandelnden dürfen nicht ausgeweitet werden. Die Patientinnen und Patienten dürfen nicht zur Offenlegung von Angaben über ihre körperliche Verfassung verpflichtet werden, die keinen Behandlungsbezug haben.

- Die Patientinnen und Patienten müssen in jedem Fall und nicht erst auf Nachfrage über erlittene Behandlungsfehler informiert werden.
- Der Gesetzentwurf sollte im Zusammenhang mit der Behandlungsdokumentation um verlässliche Vorgaben zur Absicherung des Auskunftsrechts der Patientinnen und Patienten sowie zur Archivierung und Löschung ergänzt werden.

- Der Zugang der Patientinnen und Patienten zu der sie betreffenden Behandlungsdokumentation darf nur in besonderen Ausnahmefällen eingeschränkt werden. Die in dem Entwurf vorgesehenen Beschränkungen sind zu weitgehend und unpräzise. Zudem sollte klargestellt werden, dass auch berechtigte eigene Interessen der Angehörigen einen Auskunftsanspruch begründen können.

- Der Gesetzentwurf ist um Regelungen zur Einbeziehung Dritter im Rahmen eines Behandlungsvertrages (Auftragsdatenverarbeitung) zu ergänzen.

- Regelungsbedürftig ist ferner der Umgang mit der Behandlungsdokumentation beispielsweise im Falle eines vorübergehenden Ausfalls, des Todes oder der Insolvenz des Behandelnden. Im Bereich der Heilberufe fehlt es - anders als z. B. bei den Rechtsanwälten - an einem bundesweit einheitlichen Rechtsrahmen.

\section{Digitale Agenda: Verordnung für grenzüberschreitende Verwendung elektronischer Signaturen und effektivere elektronische Identifizierung}

Als letzte der 12 Schlüsselaktionen, die in der Binnenmarktakte vorgesehen waren, hat die Europäische Kommission am 04.06.2012 neue Vorschriften vorgeschlagen, um grenzüberschreitende und sichere elektronische Transaktionen in Europa zu ermöglichen. Die vorgeschlagene Verordnung wird dafür sorgen, dass Personen und Unternehmen mit ihren eigenen nationalen elektronischen Identifizierungssystemen (eID-Systeme) öffentliche Dienste in anderen EU-Ländern benutzen können, sofern dort eine elektronische Identifizierung verwendet wird. Außerdem schafft sie einen Binnenmarkt für die grenzüberschreitende Verwendung elektronischer Signaturen (e-Signaturen) und einschlägiger Vertrauensdienste, indem sie dafür sorgt, dass diese Dienste grenzübergreifend funktionieren und den gleichen Rechtsstatus haben werden wie herkömmliche papiergestützte Verfahren. Erst dadurch wird das große Potenzial der elektronischen Auftragsvergabe („eBeschaffung “) voll zum Tragen kommen.

Der Vorschlag respektiert sowohl vorhandene nationale Identifizierungssysteme als auch die Präferenzen jener Mitgliedstaaten, die keine nationalen Identifizierungssysteme haben. Länder mit eigenem elD-System haben die Wahl, ob sie sich am europäischen System beteiligen oder nicht. Sobald ein Mitgliedstaat mitteilt, dass er sich am europaweiten System beteiligen möchte, muss er zu seinen öffentlichen Diensten den gleichen Zugang per elektronischer Identifizierung anbieten, wie ihn seine eigenen Bürger genießen.

Die vorgeschlagene Verordnung wird jedoch

- weder EU-Mitgliedstaaten zur Einführung noch deren Bürger zur Nutzung nationaler Personalausweise, elektronischer Personalausweise oder anderer elD-Lösungen verpflichten,

- kein europäisches eID-System und keine europäischen Datenbanken einführen,

- keine Zugänglichmachung persönlicher Daten für Dritte ermöglichen oder vorschreiben. 